\title{
Proteomic Translation of Chronic Granulomatous Disease (CGD)
}

\begin{abstract}
Keywords: CGD; NADPH oxidase; Mulch pneumonitis
Abstract

Chronic granulomatous disease considered as one of the congenital hereditary disease that present due to mutation in one of these following genes; CYBA, CYBB, NCF1, NCF2, or NCF4 gene in X chromosome, that lead to lack a body defense mechanisms against infections specially bacterial \& fungal infections due to absence of NADPH oxidase productions in phagocytic cells; lungs is the most common site of infections. Sometimes the causes of CGD is unknown \& we did not have a scientific explanation for this; the main aim of this study is to identify the CYBB gene SNPs change in a way to predict mutation effects of this gene at the proteomic level; through in silico tools by using sift, polyphen-2, I mutant suite-3, SNPs \& GO software prediction programs for SNPs detections. A according to these predictions tools \& their confirmations tools I found that CYBB gene SNPs mutation showed damaging predictions which was considered as clinical manifestation of this study beside this; a lots of those SNPS illustrate decreasing in protein functionality even those that were predicted benign by polyphen- 2 .
\end{abstract}

\section{Introduction}

CGD is a congenital immune deficiency disease that is genetically inherited in an X-linked manner; these means only men can be infected; also both sexes can be infected in case of autosomal recessive forms. CGD manifested by recurrent severe infections including; pneumonia, lymphadenitis, skin and hepatic abscesses, osteomyelitis and septicemia; inflammation of these tissue areas in various organs (granulomas) can result on tissue damaging. Usually infections become apparent during the first year of life; in this disease phagocytic neutrophils are unable to produce a bactericidal respiratory burst due to a deficiency of one of the proteins component of the NADPH oxidase complex [1-8].

The features of chronic granulomatous disease usually first appear in childhood, although some individuals do not show symptoms until later on in their life; they may have at least one serious bacterial or fungal infections every 3 to 4 years, especially in the lungs (pneumonia) or fungal pneumonia (mulch pneumonitis; which causes fever and shortness of breath after exposure to decaying organic materials such as mulch, hay, or dead leaves). Other common areas of infection include; the skin, liver, and lymph nodes; so the most common area of inflammation are gastrointestinal tract; ( in many cases the intestinal wall is inflamed, causing a form of inflammatory bowel disease that varies in severity but can lead to stomach pain, diarrhea, bloody stool, nausea, and vomiting) and the genitourinary tract, in addition to the stomach, colon, and rectum, as well as the mouth, throat, and skin inflammations; also, inflammation in the stomach can prevent food from passing through esophagus to the intestines (gastric outlet obstruction), leading to an inability to digest food, vomiting after eating and weight loss. In the genitourinary tract, inflammation can occur in the kidneys and bladder [2].

Inflammation of the lymph nodes (lymphadenitis) and bone marrow (osteomyelitis), which both produce immune cells,

\section{Journal of}

Proteomics \&

Computational

\section{Biology}

\author{
Hiba Siddig Ibrahim* \\ National Ribat University, Sudan \\ ${ }^{*}$ Address for Correspondence \\ Hiba Siddig Ibrahim, National Ribat University, Sudan; E-mail: \\ hibasiddig55@gmail.com \\ Submission: 27 February 2017 \\ Accepted: 10 April 2017 \\ Published: 21 April 2017 \\ Copyright: () 2017 Ibrahim HS, et al. This is an open access article \\ distributed under the Creative Commons Attribution License, which \\ permits unrestricted use, distribution, and reproduction in any medium, \\ provided the original work is properly cited.
}

can lead to further impairment of the immune system; rarely those people with chronic granulomatous disease develop autoimmune disorders. Repeated episodes of infection and inflammation reduce the life expectancy of individuals with chronic granulomatous disease; however, with the treatment, they can live until mid to late adult hood. The disease can occur in 1 in 200,000 to 250,000 people worldwide, due to mutation in the CYBA, CYBB, NCF1, NCF2, or NCF4 gene, which leads to the presence of five types of this condition. The proteins which produced from those affected genes are parts (subunits) of an enzyme complex called NADPH oxidase, that plays an essential role in the immune system, specifically in phagocytes; by production of superoxide that is used to generate other toxic substances, which play a role in killing foreign invaders and preventing them from reproducing in the body and causing illness. NADPH oxidase is also thought to regulate the activity of neutrophils, which play a critical role in adjusting the inflammatory response to optimize healing and reduce injury to the body. Beside the above, mutation in those genes can lead to production of proteins with little or no function or the productions of no protein at all [1-8].

Chronic granulomatous disease that caused by mutations in the $\mathrm{CYBB}$ gene is inherited in an $\mathrm{X}$-linked recessive pattern. The CYBB gene is located on the $\mathrm{X}$ chromosome, which is one of the two sex chromosomes. When chronic granulomatous disease is caused by CYBA, NCF1, NCF2, or NCF4 gene mutations, the condition is inherited in an autosomal recessive pattern, which means both copies of the gene in each cell have mutations [2].

Therapeutic options for CGD included prophylactic antibiotics and antifungal medications, interferon-gamma injections, and aggressive management of acute infections. Bone marrow transplantation can cure CGD, however this therapy is complex and transplant candidates and donors must be carefully selected, weighing the risks and benefits carefully. Researchers are investigating other approaches including gene therapy as a future option [1-8].

In this study I used different computational methods to identify the CYBB gene SNPs to predict mutation effects at the proteomic level. 
Citation: Ibrahim HS. Proteomic Translation of Chronic Granulomatous Disease (CGD). J Proteomics Computational Biol. 2017;3(1): 12.

ISSN: 2572-8679

Table 1: Illustrate SIFT \& Polyphen-2 predictions results.

\begin{tabular}{|c|c|c|c|c|c|c|}
\hline SNP & Organism/Build & $\begin{array}{l}\text { Amino acid } \\
\text { change }\end{array}$ & Sift score & Sift prediction & Polyphen resuit & $\begin{array}{l}\text { Polyphen } \\
\text { score }\end{array}$ \\
\hline rs137854585 & Homo_sapiens/GRCh37.74 & $\mathrm{P} 415 \mathrm{H}$ & 0 & Deleterious & Probably damaging & 1 \\
\hline rs137854585 & Homo_sapiens/GRCh37.74 & $\mathrm{P} 148 \mathrm{H}$ & 0 & Deleterious & Probably damaging & 1 \\
\hline rs 137854585 & Homo_sapiens/GRCh37.74 & P383H & 0 & Deleterious & Probably damaging & 1 \\
\hline rs137854585 & Homo_sapiens/GRCh37.74 & P415L & 0 & Deleterious & Probably damaging & 1 \\
\hline rs137854585 & Homo_sapiens/GRCh37.74 & P148L & 0 & Deleterious & Probably damaging & 1 \\
\hline rs137854585 & Homo_sapiens/GRCh37.74 & P383L & 0 & Deleterious & Probably damaging & 1 \\
\hline rs137854586 & Homo_sapiens/GRCh37.74 & G389E & 0 & Deleterious & Probably damaging & 1 \\
\hline rs137854586 & Homo_sapiens/GRCh37.74 & G122E & 0 & Deleterious & Probably damaging & 1 \\
\hline rs137854586 & Homo_sapiens/GRCh37.74 & G357E & 0 & Deleterious & Probably damaging & 1 \\
\hline rs137854586 & Homo_sapiens/GRCh37.74 & G389A & 0 & Deleterious & Probably damaging & 1 \\
\hline rs137854586 & Homo_sapiens/GRCh37.74 & G122A & 0 & Deleterious & Probably damaging & 1 \\
\hline rs137854586 & Homo_sapiens/GRCh37.74 & G357A & 0 & Deleterious & Probably damaging & 1 \\
\hline rs137854587 & Homo_sapiens/GRCh37.74 & $\mathrm{H} 209 \mathrm{Y}$ & 0 & Deleterious & Probably damaging & 1 \\
\hline rs137854587 & Homo_sapiens/GRCh37.74 & $\mathrm{H} 177 \mathrm{Y}$ & 0 & Deleterious & Probably damaging & 1 \\
\hline rs137854589 & Homo_sapiens/GRCh37.74 & $\mathrm{C} 212 \mathrm{Y}$ & 0.015 & Deleterious & Probably damaging & 1 \\
\hline rs137854589 & Homo_sapiens/GRCh37.74 & $\mathrm{C} 244 \mathrm{Y}$ & 0.034 & Deleterious & Probably damaging & 1 \\
\hline rs137854591 & Homo_sapiens/GRCh37.74 & $\mathrm{H} 101 \mathrm{R}$ & 0 & Deleterious & Probably damaging & 1 \\
\hline rs137854591 & Homo_sapiens/GRCh37.74 & $\mathrm{H} 69 \mathrm{R}$ & 0 & Deleterious & Probably damaging & 1 \\
\hline rs 137854593 & Homo_sapiens/GRCh37.74 & D500G & 0.004 & Deleterious & Probably damaging & 1 \\
\hline rs137854593 & Homo_sapiens/GRCh37.74 & D233G & 0.004 & Deleterious & Probably damaging & 1 \\
\hline rs137854593 & Homo_sapiens/GRCh37.74 & D468G & 0.005 & Deleterious & Probably damaging & 1 \\
\hline rs137854594 & Homo_sapiens/GRCh37.74 & $\mathrm{H} 101 \mathrm{Y}$ & 0 & Deleterious & Probably damaging & 1 \\
\hline rs137854594 & Homo_sapiens/GRCh37.74 & $\mathrm{H} 69 \mathrm{Y}$ & 0 & Deleterious & Probably damaging & 1 \\
\hline rs137854595 & Homo_sapiens/GRCh37.74 & $\mathrm{H} 36 \mathrm{~N}$ & 0.002 & Deleterious & Probably damaging & 0.995 \\
\hline rs137854595 & Homo_sapiens/GRCh37.74 & $\mathrm{H} 303 \mathrm{~N}$ & 0.003 & Deleterious & Probably damaging & 0.987 \\
\hline rs137854595 & Homo_sapiens/GRCh37.74 & $\mathrm{H} 271 \mathrm{~N}$ & 0.003 & Deleterious & Probably damaging & 0.996 \\
\hline rs137854596 & Homo_sapiens/GRCh37.74 & P37R & 0.002 & Deleterious & Probably damaging & 1 \\
\hline rs137854596 & Homo_sapiens/GRCh37.74 & P304R & 0.004 & Deleterious & Probably damaging & 1 \\
\hline rs137854596 & Homo_sapiens/GRCh37.74 & $\mathrm{P} 272 \mathrm{R}$ & 0.005 & Deleterious & Probably damaging & 1 \\
\hline rs139670417 & Homo_sapiens/GRCh37.74 & $\mathrm{R} 229 \mathrm{H}$ & 0.004 & Deleterious & Probably damaging & 1 \\
\hline rs139670417 & Homo_sapiens/GRCh37.74 & $\mathrm{R} 197 \mathrm{H}$ & 0.004 & Deleterious & Probably damaging & 1 \\
\hline rs140677309 & Homo_sapiens/GRCh37.74 & $\mathrm{S} 258 \mathrm{C}$ & 0.013 & Deleterious & Possibly damaging & 0.648 \\
\hline rs140677309 & Homo_sapiens/GRCh37.74 & S525C & 0.037 & Deleterious & Probably damaging & 0.965 \\
\hline rs140677309 & Homo_sapiens/GRCh37.74 & $\mathrm{S} 493 \mathrm{C}$ & 0.037 & Deleterious & Possibly damaging & 0.775 \\
\hline rs141798777 & Homo_sapiens/GRCh37.74 & L75M & 0.035 & Deleterious & Probably damaging & 1 \\
\hline rs141798777 & Homo_sapiens/GRCh37.74 & L43M & 0.036 & Deleterious & Probably damaging & 1 \\
\hline rs146275471 & Homo_sapiens/GRCh37.74 & $\mathrm{R} 198 \mathrm{Q}$ & 0.002 & Deleterious & Probably damaging & 1 \\
\hline rs146275471 & Homo_sapiens/GRCh37.74 & R166Q & 0.002 & Deleterious & Probably damaging & 0.997 \\
\hline rs151344453 & Homo_sapiens/GRCh37.74 & Y41D & 0.031 & Deleterious & Probably damaging & 1 \\
\hline rs151344454 & Homo_sapiens/GRCh37.74 & C537R & 0 & Deleterious & Probably damaging & 1 \\
\hline rs151344454 & Homo_sapiens/GRCh37.74 & $\mathrm{C} 270 \mathrm{R}$ & 0 & Deleterious & Probably damaging & 1 \\
\hline
\end{tabular}


Citation: Ibrahim HS. Proteomic Translation of Chronic Granulomatous Disease (CGD). J Proteomics Computational Biol. 2017;3(1): 12.

ISSN: $2572-8679$

\begin{tabular}{|c|c|c|c|c|c|c|}
\hline rs151344454 & Homo_sapiens/GRCh37.74 & C505R & 0 & Deleterious & Probably damaging & 1 \\
\hline rs151344456 & Homo_sapiens/GRCh37.74 & R54S & 0.001 & Deleterious & Probably damaging & 0.996 \\
\hline rs151344456 & Homo_sapiens/GRCh37.74 & $\mathrm{R} 22 \mathrm{~S}$ & 0.001 & Deleterious & Probably damaging & 0.997 \\
\hline rs151344457 & Homo_sapiens/GRCh37.74 & C59R & 0.003 & Deleterious & Probably damaging & 0.999 \\
\hline rs151344457 & Homo_sapiens/GRCh37.74 & $\mathrm{C} 27 \mathrm{R}$ & 0.004 & Deleterious & Probably damaging & 0.999 \\
\hline rs151344458 & Homo_sapiens/GRCh37.74 & H119R & 0 & Deleterious & Probably damaging & 1 \\
\hline rs151344458 & Homo_sapiens/GRCh37.74 & H87R & 0 & Deleterious & Probably damaging & 1 \\
\hline rs151344459 & Homo_sapiens/GRCh37.74 & H209Q & 0 & Deleterious & Probably damaging & 1 \\
\hline rs151344459 & Homo_sapiens/GRCh37.74 & $\mathrm{H} 177 \mathrm{Q}$ & 0 & Deleterious & Probably damaging & 1 \\
\hline rs151344460 & Homo_sapiens/GRCh37.74 & $\mathrm{H} 222 \mathrm{~N}$ & 0 & Deleterious & Probably damaging & 0.998 \\
\hline rs151344460 & Homo_sapiens/GRCh37.74 & $\mathrm{H} 190 \mathrm{~N}$ & 0 & Deleterious & Probably damaging & 1 \\
\hline rs151344460 & Homo_sapiens/GRCh37.74 & $\mathrm{H} 222 \mathrm{Y}$ & 0 & Deleterious & Probably damaging & 1 \\
\hline rs151344460 & Homo_sapiens/GRCh37.74 & H190Y & 0 & Deleterious & Probably damaging & 1 \\
\hline rs151344462 & Homo_sapiens/GRCh37.74 & $\mathrm{H} 222 \mathrm{R}$ & 0 & Deleterious & Probably damaging & 1 \\
\hline rs151344462 & Homo_sapiens/GRCh37.74 & H190R & 0 & Deleterious & Probably damaging & 1 \\
\hline rs151344465 & Homo_sapiens/GRCh37.74 & $\mathrm{C} 212 \mathrm{R}$ & 0.013 & Deleterious & Probably damaging & 1 \\
\hline rs151344465 & Homo_sapiens/GRCh37.74 & C244R & 0.02 & Deleterious & Probably damaging & 1 \\
\hline rs151344466 & Homo_sapiens/GRCh37.74 & $\mathrm{E} 42 \mathrm{~K}$ & 0.002 & Deleterious & Probably damaging & 1 \\
\hline rs151344466 & Homo_sapiens/GRCh37.74 & E309K & 0.003 & Deleterious & Probably damaging & 1 \\
\hline rs151344466 & Homo_sapiens/GRCh37.74 & E277K & 0.003 & Deleterious & Probably damaging & 1 \\
\hline rs151344467 & Homo_sapiens/GRCh37.74 & G322E & 0 & Deleterious & Probably damaging & 1 \\
\hline rs151344467 & Homo_sapiens/GRCh37.74 & G55E & 0 & Deleterious & Probably damaging & 1 \\
\hline rs151344467 & Homo_sapiens/GRCh37.74 & G290E & 0 & Deleterious & Probably damaging & 1 \\
\hline rs151344468 & Homo_sapiens/GRCh37.74 & I325F & 0.002 & Deleterious & Probably damaging & 0.99 \\
\hline rs151344468 & Homo_sapiens/GRCh37.74 & $158 \mathrm{~F}$ & 0.002 & Deleterious & Probably damaging & 0.987 \\
\hline rs151344468 & Homo_sapiens/GRCh37.74 & I293F & 0.002 & Deleterious & Probably damaging & 0.993 \\
\hline rs151344469 & Homo_sapiens/GRCh37.74 & $\mathrm{S} 66 \mathrm{P}$ & 0.003 & Deleterious & Probably damaging & 1 \\
\hline rs151344469 & Homo_sapiens/GRCh37.74 & S333P & 0.004 & Deleterious & Probably damaging & 1 \\
\hline rs151344469 & Homo_sapiens/GRCh37.74 & S301P & 0.004 & Deleterious & Probably damaging & 1 \\
\hline rs151344470 & Homo_sapiens/GRCh37.74 & P339H & 0 & Deleterious & Probably damaging & 0.994 \\
\hline rs151344470 & Homo_sapiens/GRCh37.74 & $\mathrm{P} 72 \mathrm{H}$ & 0 & Deleterious & Probably damaging & 1 \\
\hline rs151344470 & Homo_sapiens/GRCh37.74 & P307H & 0 & Deleterious & Probably damaging & 0.999 \\
\hline rs151344471 & Homo_sapiens/GRCh37.74 & R89P & 0.001 & Deleterious & Probably damaging & 1 \\
\hline rs151344471 & Homo_sapiens/GRCh37.74 & R356P & 0.002 & Deleterious & Probably damaging & 1 \\
\hline rs151344471 & Homo_sapiens/GRCh37.74 & R324P & 0.002 & Deleterious & Probably damaging & 1 \\
\hline rs151344472 & Homo_sapiens/GRCh37.74 & M138R & 0.002 & Deleterious & Probably damaging & 0.997 \\
\hline rs151344472 & Homo_sapiens/GRCh37.74 & M373R & 0.002 & Deleterious & Probably damaging & 0.999 \\
\hline rs151344472 & Homo_sapiens/GRCh37.74 & M405R & 0.003 & Deleterious & Probably damaging & 0.999 \\
\hline rs151344473 & Homo_sapiens/GRCh37.74 & G408R & 0.001 & Deleterious & Probably damaging & 1 \\
\hline rs151344473 & Homo_sapiens/GRCh37.74 & G141R & 0.001 & Deleterious & Probably damaging & 1 \\
\hline rs151344473 & Homo_sapiens/GRCh37.74 & G376R & 0.001 & Deleterious & Probably damaging & 1 \\
\hline rs151344474 & Homo_sapiens/GRCh37.74 & G408E & 0.001 & Deleterious & Probably damaging & 1 \\
\hline rs151344474 & Homo_sapiens/GRCh37.74 & G141E & 0.001 & Deleterious & Probably damaging & 1 \\
\hline rs 151344474 & Homo_sapiens/GRCh37.74 & G376E & 0.001 & Deleterious & Probably damaging & 1 \\
\hline rs151344475 & Homo_sapiens/GRCh37.74 & S422P & 0.006 & Deleterious & Probably damaging & 1 \\
\hline
\end{tabular}


Citation: Ibrahim HS. Proteomic Translation of Chronic Granulomatous Disease (CGD). J Proteomics Computational Biol. $2017 ; 3(1): 12$.

ISSN: $2572-8679$

\begin{tabular}{|c|c|c|c|c|c|c|}
\hline rs151344475 & Homo_sapiens/GRCh37.74 & S390P & 0.007 & Deleterious & Probably damaging & 1 \\
\hline rs151344475 & Homo_sapiens/GRCh37.74 & S155P & 0.013 & Deleterious & Probably damaging & 1 \\
\hline rs151344477 & Homo_sapiens/GRCh37.74 & W516C & 0 & Deleterious & Probably damaging & 1 \\
\hline rs151344477 & Homo_sapiens/GRCh37.74 & W249C & 0 & Deleterious & Probably damaging & 1 \\
\hline rs151344477 & Homo_sapiens/GRCh37.74 & W484C & 0 & Deleterious & Probably damaging & 1 \\
\hline rs151344478 & Homo_sapiens/GRCh37.74 & V534D & 0 & Deleterious & Probably damaging & 0.998 \\
\hline rs151344478 & Homo_sapiens/GRCh37.74 & V267D & 0 & Deleterious & Probably damaging & 0.998 \\
\hline rs151344478 & Homo_sapiens/GRCh37.74 & V502D & 0 & Deleterious & Probably damaging & 0.999 \\
\hline rs151344479 & Homo_sapiens/GRCh37.74 & R54M & 0 & Deleterious & Probably damaging & 1 \\
\hline rs151344479 & Homo_sapiens/GRCh37.74 & $\mathrm{R} 22 \mathrm{M}$ & 0 & Deleterious & Probably damaging & 1 \\
\hline rs151344480 & Homo_sapiens/GRCh37.74 & A55D & 0.001 & Deleterious & Probably damaging & 1 \\
\hline rs151344480 & Homo_sapiens/GRCh37.74 & A23D & 0.002 & Deleterious & Probably damaging & 1 \\
\hline rs151344481 & Homo_sapiens/GRCh37.74 & A57E & 0.001 & Deleterious & Probably damaging & 1 \\
\hline rs151344481 & Homo_sapiens/GRCh37.74 & A25E & 0.001 & Deleterious & Probably damaging & 1 \\
\hline rs151344482 & Homo_sapiens/GRCh37.74 & H209R & 0 & Deleterious & Probably damaging & 1 \\
\hline rs151344482 & Homo_sapiens/GRCh37.74 & H177R & 0 & Deleterious & Probably damaging & 1 \\
\hline rs151344484 & Homo_sapiens/GRCh37.74 & H338Y & 0.001 & Deleterious & Probably damaging & 1 \\
\hline rs151344484 & Homo_sapiens/GRCh37.74 & $\mathrm{H} 71 \mathrm{Y}$ & 0.001 & Deleterious & Probably damaging & 0.999 \\
\hline rs151344484 & Homo_sapiens/GRCh37.74 & H306Y & 0.001 & Deleterious & Probably damaging & 1 \\
\hline rs151344485 & Homo_sapiens/GRCh37.74 & S312F & 0.001 & Deleterious & Probably damaging & 1 \\
\hline rs151344485 & Homo_sapiens/GRCh37.74 & S344F & 0.004 & Deleterious & Probably damaging & 1 \\
\hline rs151344485 & Homo_sapiens/GRCh37.74 & S77F & 0.005 & Deleterious & Probably damaging & 1 \\
\hline rs151344486 & Homo_sapiens/GRCh37.74 & L420P & 0 & Deleterious & Probably damaging & 1 \\
\hline rs151344486 & Homo_sapiens/GRCh37.74 & L153P & 0 & Deleterious & Probably damaging & 1 \\
\hline rs151344486 & Homo_sapiens/GRCh37.74 & L388P & 0 & Deleterious & Probably damaging & 1 \\
\hline rs151344487 & Homo_sapiens/GRCh37.74 & W516R & 0 & Deleterious & Probably damaging & 0.996 \\
\hline rs151344487 & Homo_sapiens/GRCh37.74 & W249R & 0 & Deleterious & Probably damaging & 0.999 \\
\hline rs 151344487 & Homo_sapiens/GRCh37.74 & W484R & 0 & Deleterious & Probably damaging & 0.999 \\
\hline rs151344488 & Homo_sapiens/GRCh37.74 & C59W & 0.001 & Deleterious & Probably damaging & 1 \\
\hline rs151344488 & Homo_sapiens/GRCh37.74 & $\mathrm{C} 27 \mathrm{~W}$ & 0.027 & Deleterious & Probably damaging & 1 \\
\hline rs151344489 & Homo_sapiens/GRCh37.74 & T307P & 0.002 & Deleterious & Probably damaging & 0.999 \\
\hline rs151344489 & Homo_sapiens/GRCh37.74 & $\mathrm{T} 40 \mathrm{P}$ & 0.002 & Deleterious & Probably damaging & 0.997 \\
\hline rs151344489 & Homo_sapiens/GRCh37.74 & T275P & 0.002 & Deleterious & Probably damaging & 0.999 \\
\hline rs151344490 & Homo_sapiens/GRCh37.74 & L505R & 0.001 & Deleterious & Probably damaging & 0.999 \\
\hline rs151344490 & Homo_sapiens/GRCh37.74 & L238R & 0.001 & Deleterious & Probably damaging & 1 \\
\hline rs151344490 & Homo_sapiens/GRCh37.74 & L473R & 0.001 & Deleterious & Probably damaging & 1 \\
\hline rs151344491 & Homo_sapiens/GRCh37.74 & G179R & 0 & Deleterious & Probably damaging & 1 \\
\hline rs151344491 & Homo_sapiens/GRCh37.74 & G147R & 0 & Deleterious & Probably damaging & 1 \\
\hline rs151344492 & Homo_sapiens/GRCh37.74 & L546P & 0 & Deleterious & Probably damaging & 1 \\
\hline rs151344492 & Homo_sapiens/GRCh37.74 & L279P & 0 & Deleterious & Probably damaging & 1 \\
\hline rs151344492 & Homo_sapiens/GRCh37.74 & L514P & 0 & Deleterious & Probably damaging & 1 \\
\hline rs151344493 & Homo_sapiens/GRCh37.74 & S193F & 0.001 & Deleterious & Probably damaging & 1 \\
\hline rs151344493 & Homo_sapiens/GRCh37.74 & S161F & 0.002 & Deleterious & Probably damaging & 1 \\
\hline rs151344495 & Homo_sapiens/GRCh37.74 & L342Q & 0.001 & Deleterious & Probably damaging & 0.999 \\
\hline rs151344495 & Homo_sapiens/GRCh37.74 & L75Q & 0.001 & Deleterious & Probably damaging & 1 \\
\hline rs151344495 & Homo_sapiens/GRCh37.74 & L310Q & 0.001 & Deleterious & Probably damaging & 1 \\
\hline
\end{tabular}


Citation: Ibrahim HS. Proteomic Translation of Chronic Granulomatous Disease (CGD). J Proteomics Computational Biol. $2017 ; 3(1): 12$.

ISSN: $2572-8679$

\begin{tabular}{|c|c|c|c|c|c|c|}
\hline rs151344496 & Homo_sapiens/GRCh37.74 & F205I & 0 & Deleterious & Probably damaging & 1 \\
\hline rs151344496 & Homo_sapiens/GRCh37.74 & F173I & 0 & Deleterious & Probably damaging & 1 \\
\hline rs151344497 & Homo_sapiens/GRCh37.74 & T178P & 0.001 & Deleterious & Probably damaging & 1 \\
\hline rs151344497 & Homo_sapiens/GRCh37.74 & T146P & 0.001 & Deleterious & Probably damaging & 1 \\
\hline rs151344498 & Homo_sapiens/GRCh37.74 & Q231P & 0.006 & Deleterious & Probably damaging & 0.992 \\
\hline rs151344498 & Homo_sapiens/GRCh37.74 & Q199P & 0.006 & Deleterious & Probably damaging & 0.997 \\
\hline rs200614534 & Homo_sapiens/GRCh37.74 & V407A & 0.001 & Deleterious & Probably damaging & 0.995 \\
\hline rs200614534 & Homo_sapiens/GRCh37.74 & V140A & 0.002 & Deleterious & Probably damaging & 0.988 \\
\hline rs200614534 & Homo_sapiens/GRCh37.74 & V375A & 0.002 & Deleterious & Probably damaging & 0.994 \\
\hline rs267606451 & Homo_sapiens/GRCh37.74 & S66F & 0 & Deleterious & Probably damaging & 1 \\
\hline rs267606451 & Homo_sapiens/GRCh37.74 & S333F & 0.001 & Deleterious & Probably damaging & 1 \\
\hline rs267606451 & Homo_sapiens/GRCh37.74 & S301F & 0.001 & Deleterious & Probably damaging & 1 \\
\hline
\end{tabular}

Table 2: Illustrate I-mutant 3 prediction results for protein activity.

\begin{tabular}{|c|c|c|c|c|c|c|c|}
\hline SNP & Organism/Build & $\begin{array}{l}\text { Amino Acid } \\
\text { Change }\end{array}$ & WT & MT & DDG & RI & I Mutant Result \\
\hline rs137854585 & Homo_sapiens/GRCh37.74 & $\mathrm{P} 415 \mathrm{H}$ & $\mathrm{P}$ & $\mathrm{H}$ & -1.69 & 9 & Decrease \\
\hline rs137854585 & Homo_sapiens/GRCh37.74 & $\mathrm{P} 148 \mathrm{H}$ & $\mathrm{P}$ & $\mathrm{H}$ & -1.69 & 9 & Decrease \\
\hline rs137854585 & Homo_sapiens/GRCh37.74 & P383H & $\mathrm{P}$ & $\mathrm{H}$ & -1.69 & 9 & Decrease \\
\hline rs137854585 & Homo_sapiens/GRCh37.74 & P415L & $\mathrm{P}$ & $\mathrm{L}$ & -0.77 & 6 & Decrease \\
\hline rs137854585 & Homo_sapiens/GRCh37.74 & P148L & $\mathrm{P}$ & $L$ & -0.77 & 6 & Decrease \\
\hline rs137854585 & Homo_sapiens/GRCh37.74 & P383L & $\mathrm{P}$ & $\mathrm{L}$ & -0.77 & 6 & Decrease \\
\hline rs137854586 & Homo_sapiens/GRCh37.74 & G389E & G & $E$ & -0.69 & 3 & Decrease \\
\hline rs137854586 & Homo_sapiens/GRCh37.74 & G122E & G & $E$ & -0.69 & 3 & Decrease \\
\hline rs137854586 & Homo_sapiens/GRCh37.74 & G357E & G & $E$ & -0.69 & 3 & Decrease \\
\hline rs137854586 & Homo_sapiens/GRCh37.74 & G389A & G & A & -0.93 & 8 & Decrease \\
\hline rs137854586 & Homo_sapiens/GRCh37.74 & G122A & G & A & -0.93 & 8 & Decrease \\
\hline rs137854586 & Homo_sapiens/GRCh37.74 & G357A & G & A & -0.93 & 8 & Decrease \\
\hline rs137854587 & Homo_sapiens/GRCh37.74 & H209Y & $\mathrm{H}$ & $\mathrm{Y}$ & 0.49 & 8 & Increase \\
\hline rs137854587 & Homo_sapiens/GRCh37.74 & $\mathrm{H} 177 \mathrm{Y}$ & $\mathrm{H}$ & $\mathrm{Y}$ & 0.49 & 8 & Increase \\
\hline rs137854589 & Homo_sapiens/GRCh37.74 & C212Y & C & $\mathrm{Y}$ & -0.15 & 0 & Decrease \\
\hline rs137854589 & Homo_sapiens/GRCh37.74 & C244Y & C & Y & -0.15 & 0 & Decrease \\
\hline rs137854591 & Homo_sapiens/GRCh37.74 & H101R & $\mathrm{H}$ & $\mathrm{R}$ & -0.09 & 2 & Decrease \\
\hline rs137854591 & Homo_sapiens/GRCh37.74 & H69R & $\mathrm{H}$ & $\mathrm{R}$ & -0.09 & 2 & Decrease \\
\hline rs137854593 & Homo_sapiens/GRCh37.74 & D500G & $\mathrm{D}$ & G & -0.57 & 2 & Decrease \\
\hline rs137854593 & Homo_sapiens/GRCh37.74 & D233G & $\mathrm{D}$ & G & -0.57 & 2 & Decrease \\
\hline rs137854593 & Homo_sapiens/GRCh37.74 & D468G & $\mathrm{D}$ & G & -0.57 & 2 & Decrease \\
\hline rs137854594 & Homo_sapiens/GRCh37.74 & H101Y & $\mathrm{H}$ & Y & 0.24 & 3 & Increase \\
\hline rs137854594 & Homo_sapiens/GRCh37.74 & $\mathrm{H} 69 \mathrm{Y}$ & $\mathrm{H}$ & $\mathrm{Y}$ & 0.24 & 3 & Increase \\
\hline rs137854595 & Homo_sapiens/GRCh37.74 & $\mathrm{H} 36 \mathrm{~N}$ & $\mathrm{H}$ & $\mathrm{N}$ & -0.38 & 3 & Decrease \\
\hline rs137854595 & Homo_sapiens/GRCh37.74 & $\mathrm{H} 303 \mathrm{~N}$ & $\mathrm{H}$ & $\mathrm{N}$ & -0.38 & 3 & Decrease \\
\hline rs137854595 & Homo_sapiens/GRCh37.74 & $\mathrm{H} 271 \mathrm{~N}$ & $\mathrm{H}$ & $\mathrm{N}$ & -0.38 & 3 & Decrease \\
\hline rs137854596 & Homo_sapiens/GRCh37.74 & P37R & $\mathrm{P}$ & $\mathrm{R}$ & -0.65 & 4 & Decrease \\
\hline rs137854596 & Homo_sapiens/GRCh37.74 & P304R & $\mathrm{P}$ & $\mathrm{R}$ & -0.65 & 4 & Decrease \\
\hline rs137854596 & Homo_sapiens/GRCh37.74 & P272R & $\mathrm{P}$ & $\mathrm{R}$ & -0.65 & 4 & Decrease \\
\hline rs139670417 & Homo_sapiens/GRCh37.74 & $\mathrm{R} 229 \mathrm{H}$ & $\mathrm{R}$ & $\mathrm{H}$ & -1.07 & 8 & Decrease \\
\hline rs139670417 & Homo_sapiens/GRCh37.74 & R197H & $\mathrm{R}$ & $\mathrm{H}$ & -1.07 & 8 & Decrease \\
\hline
\end{tabular}


Citation: Ibrahim HS. Proteomic Translation of Chronic Granulomatous Disease (CGD). J Proteomics Computational Biol. 2017;3(1): 12.

ISSN: 2572-8679

\begin{tabular}{|c|c|c|c|c|c|c|c|}
\hline rs140677309 & Homo_sapiens/GRCh37.74 & S258C & $\mathrm{s}$ & C & -0.54 & 3 & Decrease \\
\hline rs140677309 & Homo_sapiens/GRCh37.74 & S525C & $\mathrm{s}$ & C & -0.54 & 3 & Decrease \\
\hline rs140677309 & Homo_sapiens/GRCh37.74 & S493C & $\mathrm{s}$ & C & -0.54 & 3 & Decrease \\
\hline rs141798777 & Homo_sapiens/GRCh37.74 & L75M & $\mathrm{L}$ & M & -1.09 & 4 & Decrease \\
\hline rs141798777 & Homo_sapiens/GRCh37.74 & L43M & $\mathrm{L}$ & M & -1.09 & 4 & Decrease \\
\hline rs146275471 & Homo_sapiens/GRCh37.74 & R198Q & $\mathrm{R}$ & Q & -1.22 & 8 & Decrease \\
\hline rs146275471 & Homo_sapiens/GRCh37.74 & R166Q & $\mathrm{R}$ & Q & -1.22 & 8 & Decrease \\
\hline rs151344453 & Homo_sapiens/GRCh37.74 & Y41D & $\mathrm{Y}$ & D & -1.25 & 4 & Decrease \\
\hline rs151344454 & Homo_sapiens/GRCh37.74 & C537R & C & $\mathrm{R}$ & -0.31 & 4 & Decrease \\
\hline rs151344454 & Homo_sapiens/GRCh37.74 & C270R & C & $\mathrm{R}$ & -0.31 & 4 & Decrease \\
\hline rs151344454 & Homo_sapiens/GRCh37.74 & C505R & C & $\mathrm{R}$ & -0.31 & 4 & Decrease \\
\hline rs151344456 & Homo_sapiens/GRCh37.74 & R54S & $\mathrm{R}$ & $\mathrm{s}$ & -0.66 & 7 & Decrease \\
\hline rs151344456 & Homo_sapiens/GRCh37.74 & R22S & $\mathrm{R}$ & $\mathrm{s}$ & -0.66 & 7 & Decrease \\
\hline rs151344457 & Homo_sapiens/GRCh37.74 & C59R & C & $\mathrm{R}$ & -0.25 & 1 & Decrease \\
\hline rs151344457 & Homo_sapiens/GRCh37.74 & C27R & C & $\mathrm{R}$ & -0.25 & 1 & Decrease \\
\hline rs151344458 & Homo_sapiens/GRCh37.74 & $\mathrm{H} 119 \mathrm{R}$ & $\mathrm{H}$ & $\mathrm{R}$ & -0.02 & 4 & Decrease \\
\hline rs151344458 & Homo_sapiens/GRCh37.74 & $\mathrm{H} 87 \mathrm{R}$ & $\mathrm{H}$ & $\mathrm{R}$ & -0.02 & 4 & Decrease \\
\hline rs151344459 & Homo_sapiens/GRCh37.74 & H209Q & $\mathrm{H}$ & Q & -0.15 & 3 & Decrease \\
\hline rs151344459 & Homo_sapiens/GRCh37.74 & $\mathrm{H} 177 \mathrm{Q}$ & $\mathrm{H}$ & Q & -0.15 & 3 & Decrease \\
\hline rs151344460 & Homo_sapiens/GRCh37.74 & $\mathrm{H} 222 \mathrm{~N}$ & $\mathrm{H}$ & $\mathrm{N}$ & -0.64 & 3 & Decrease \\
\hline rs151344460 & Homo_sapiens/GRCh37.74 & $\mathrm{H} 190 \mathrm{~N}$ & $\mathrm{H}$ & $\mathrm{N}$ & -0.64 & 3 & Decrease \\
\hline rs151344460 & Homo_sapiens/GRCh37.74 & $\mathrm{H} 222 \mathrm{Y}$ & $\mathrm{H}$ & Y & 0.15 & 4 & Increase \\
\hline rs151344460 & Homo_sapiens/GRCh37.74 & H190Y & $\mathrm{H}$ & Y & 0.15 & 4 & Increase \\
\hline rs151344462 & Homo_sapiens/GRCh37.74 & $\mathrm{H} 222 \mathrm{R}$ & $\mathrm{H}$ & $\mathrm{R}$ & -0.16 & 4 & Decrease \\
\hline rs151344462 & Homo_sapiens/GRCh37.74 & H190R & H & $\mathrm{R}$ & -0.16 & 4 & Decrease \\
\hline rs151344465 & Homo_sapiens/GRCh37.74 & $\mathrm{C} 212 \mathrm{R}$ & C & $\mathrm{R}$ & -0.16 & 2 & Decrease \\
\hline rs151344465 & Homo_sapiens/GRCh37.74 & C244R & C & $\mathrm{R}$ & -0.16 & 2 & Decrease \\
\hline rs151344466 & Homo_sapiens/GRCh37.74 & $\mathrm{E} 42 \mathrm{~K}$ & $\mathrm{E}$ & $\mathrm{K}$ & -0.35 & 7 & Decrease \\
\hline rs151344466 & Homo_sapiens/GRCh37.74 & E309K & E & $\mathrm{K}$ & -0.35 & 7 & Decrease \\
\hline rs151344466 & Homo_sapiens/GRCh37.74 & E277K & E & $\mathrm{K}$ & -0.35 & 7 & Decrease \\
\hline rs151344467 & Homo_sapiens/GRCh37.74 & G322E & G & E & -0.8 & 4 & Decrease \\
\hline rs151344467 & Homo_sapiens/GRCh37.74 & G55E & G & E & -0.8 & 4 & Decrease \\
\hline rs151344467 & Homo_sapiens/GRCh37.74 & G290E & G & E & -0.8 & 4 & Decrease \\
\hline rs151344468 & Homo_sapiens/GRCh37.74 & I325F & 1 & $\mathrm{~F}$ & -1.19 & 4 & Decrease \\
\hline rs151344468 & Homo_sapiens/GRCh37.74 & $158 \mathrm{~F}$ & 1 & $\mathrm{~F}$ & -1.19 & 4 & Decrease \\
\hline rs151344468 & Homo_sapiens/GRCh37.74 & I293F & 1 & $\mathrm{~F}$ & -1.19 & 4 & Decrease \\
\hline rs151344469 & Homo_sapiens/GRCh37.74 & S66P & $\mathrm{s}$ & $P$ & -0.18 & 0 & Decrease \\
\hline rs151344469 & Homo_sapiens/GRCh37.74 & S333P & $\mathrm{s}$ & $\mathrm{P}$ & -0.18 & 0 & Decrease \\
\hline rs151344469 & Homo_sapiens/GRCh37.74 & S301P & $\mathrm{s}$ & $P$ & -0.18 & 0 & Decrease \\
\hline rs151344470 & Homo_sapiens/GRCh37.74 & $\mathrm{P} 339 \mathrm{H}$ & $\mathrm{P}$ & $\mathrm{H}$ & -1.32 & 7 & Decrease \\
\hline rs151344470 & Homo_sapiens/GRCh37.74 & $\mathrm{P} 72 \mathrm{H}$ & $P$ & $\mathrm{H}$ & -1.32 & 7 & Decrease \\
\hline rs151344470 & Homo_sapiens/GRCh37.74 & $\mathrm{P} 307 \mathrm{H}$ & $P$ & H & -1.32 & 7 & Decrease \\
\hline rs151344471 & Homo_sapiens/GRCh37.74 & R89P & $\mathrm{R}$ & $P$ & -0.66 & 6 & Decrease \\
\hline rs151344471 & Homo_sapiens/GRCh37.74 & R356P & $\mathrm{R}$ & $\mathrm{P}$ & -0.66 & 6 & Decrease \\
\hline rs151344471 & Homo_sapiens/GRCh37.74 & R324P & $\mathrm{R}$ & $P$ & -0.66 & 6 & Decrease \\
\hline rs151344472 & Homo_sapiens/GRCh37.74 & M138R & M & $\mathrm{R}$ & -0.62 & 2 & Decrease \\
\hline
\end{tabular}


Citation: Ibrahim HS. Proteomic Translation of Chronic Granulomatous Disease (CGD). J Proteomics Computational Biol. 2017;3(1): 12.

ISSN: 2572-8679

\begin{tabular}{|c|c|c|c|c|c|c|c|}
\hline rs151344472 & Homo_sapiens/GRCh37.74 & M373R & M & $\mathrm{R}$ & -0.62 & 2 & Decrease \\
\hline rs151344472 & Homo_sapiens/GRCh37.74 & M405R & M & $\mathrm{R}$ & -0.62 & 2 & Decrease \\
\hline rs151344473 & Homo_sapiens/GRCh37.74 & G408R & G & $\mathrm{R}$ & -0.51 & 3 & Decrease \\
\hline rs151344473 & Homo_sapiens/GRCh37.74 & G141R & G & $\mathrm{R}$ & -0.51 & 3 & Decrease \\
\hline rs151344473 & Homo_sapiens/GRCh37.74 & G376R & G & $\mathrm{R}$ & -0.51 & 3 & Decrease \\
\hline rs151344474 & Homo_sapiens/GRCh37.74 & G408E & G & E & -0.54 & 1 & Increase \\
\hline rs151344474 & Homo_sapiens/GRCh37.74 & G141E & G & E & -0.54 & 1 & Increase \\
\hline rs151344474 & Homo_sapiens/GRCh37.74 & G376E & G & E & -0.54 & 1 & Increase \\
\hline rs151344475 & Homo_sapiens/GRCh37.74 & S422P & $\mathrm{s}$ & $\mathrm{P}$ & -0.31 & 1 & Increase \\
\hline rs151344475 & Homo_sapiens/GRCh37.74 & S390P & $\mathrm{s}$ & $\mathrm{P}$ & -0.31 & 1 & Increase \\
\hline rs151344475 & Homo_sapiens/GRCh37.74 & S155P & $\mathrm{s}$ & $\mathrm{P}$ & -0.31 & 1 & Increase \\
\hline rs151344477 & Homo_sapiens/GRCh37.74 & W516C & W & C & -1.77 & 9 & Decrease \\
\hline rs151344477 & Homo_sapiens/GRCh37.74 & W249C & W & c & -1.77 & 9 & Decrease \\
\hline rs151344477 & Homo_sapiens/GRCh37.74 & W484C & W & C & -1.77 & 9 & Decrease \\
\hline rs151344478 & Homo_sapiens/GRCh37.74 & V534D & v & D & -1.49 & 9 & Decrease \\
\hline rs151344478 & Homo_sapiens/GRCh37.74 & V267D & v & D & -1.49 & 9 & Decrease \\
\hline rs151344478 & Homo_sapiens/GRCh37.74 & V502D & $\mathrm{v}$ & D & -1.49 & 9 & Decrease \\
\hline rs151344479 & Homo_sapiens/GRCh37.74 & R54M & $\mathrm{R}$ & M & -0.2 & 1 & Decrease \\
\hline rs151344479 & Homo_sapiens/GRCh37.74 & R22M & $\mathrm{R}$ & M & -0.2 & 1 & Decrease \\
\hline rs151344480 & Homo_sapiens/GRCh37.74 & A55D & A & $\mathrm{D}$ & -0.58 & 6 & Decrease \\
\hline rs151344480 & Homo_sapiens/GRCh37.74 & A23D & A & D & -0.58 & 6 & Decrease \\
\hline rs151344481 & Homo_sapiens/GRCh37.74 & A57E & A & E & -0.36 & 4 & Decrease \\
\hline rs151344481 & Homo_sapiens/GRCh37.74 & A25E & A & E & -0.36 & 4 & Decrease \\
\hline rs151344482 & Homo_sapiens/GRCh37.74 & $\mathrm{H} 209 \mathrm{R}$ & $\mathrm{H}$ & $\mathrm{R}$ & 0.12 & 1 & Decrease \\
\hline rs151344482 & Homo_sapiens/GRCh37.74 & H177R & $\mathrm{H}$ & $\mathrm{R}$ & 0.12 & 1 & Decrease \\
\hline rs151344484 & Homo_sapiens/GRCh37.74 & $\mathrm{H} 338 \mathrm{Y}$ & $\mathrm{H}$ & $\mathrm{Y}$ & 0.47 & 7 & Increase \\
\hline rs151344484 & Homo_sapiens/GRCh37.74 & $\mathrm{H} 71 \mathrm{Y}$ & $\mathrm{H}$ & $\mathrm{Y}$ & 0.47 & 7 & Increase \\
\hline rs151344484 & Homo_sapiens/GRCh37.74 & $\mathrm{H} 306 \mathrm{Y}$ & $\mathrm{H}$ & Y & 0.47 & 7 & Increase \\
\hline rs151344485 & Homo_sapiens/GRCh37.74 & S312F & $\mathrm{s}$ & $\mathrm{F}$ & 0.4 & 5 & Increase \\
\hline rs151344485 & Homo_sapiens/GRCh37.74 & S344F & $\mathrm{s}$ & $\mathrm{F}$ & 0.4 & 5 & Increase \\
\hline rs151344485 & Homo_sapiens/GRCh37.74 & S77F & $\mathrm{s}$ & $\mathrm{F}$ & 0.4 & 5 & Increase \\
\hline rs151344486 & Homo_sapiens/GRCh37.74 & L420P & L & $\mathrm{P}$ & -1.64 & 7 & Decrease \\
\hline rs151344486 & Homo_sapiens/GRCh37.74 & L153P & L & $P$ & -1.64 & 7 & Decrease \\
\hline rs151344486 & Homo_sapiens/GRCh37.74 & L388P & L & $P$ & -1.64 & 7 & Decrease \\
\hline rs151344487 & Homo_sapiens/GRCh37.74 & W516R & W & $\mathrm{R}$ & -1.3 & 9 & Decrease \\
\hline rs151344487 & Homo_sapiens/GRCh37.74 & W249R & W & $\mathrm{R}$ & -1.3 & 9 & Decrease \\
\hline rs151344487 & Homo_sapiens/GRCh37.74 & W484R & W & $\mathrm{R}$ & -1.3 & 9 & Decrease \\
\hline rs151344488 & Homo_sapiens/GRCh37.74 & C59W & C & W & -0.14 & 2 & Decrease \\
\hline rs151344488 & Homo_sapiens/GRCh37.74 & $\mathrm{C} 27 \mathrm{~W}$ & C & W & -0.14 & 2 & Decrease \\
\hline rs151344489 & Homo_sapiens/GRCh37.74 & T307P & $\mathrm{T}$ & $\mathrm{P}$ & -0.59 & 6 & Decrease \\
\hline rs151344489 & Homo_sapiens/GRCh37.74 & T40P & $\mathrm{T}$ & $\mathrm{P}$ & -0.59 & 6 & Decrease \\
\hline rs151344489 & Homo_sapiens/GRCh37.74 & T275P & $\mathrm{T}$ & $\mathrm{P}$ & -0.59 & 6 & Decrease \\
\hline rs151344490 & Homo_sapiens/GRCh37.74 & L505R & $\mathrm{L}$ & $\mathrm{R}$ & -1.63 & 8 & Decrease \\
\hline rs151344490 & Homo_sapiens/GRCh37.74 & L238R & L & $\mathrm{R}$ & -1.63 & 8 & Decrease \\
\hline rs151344490 & Homo_sapiens/GRCh37.74 & L473R & L & $\mathrm{R}$ & -1.63 & 8 & Decrease \\
\hline rs151344491 & Homo_sapiens/GRCh37.74 & G179R & G & $\mathrm{R}$ & -0.44 & 5 & Decrease \\
\hline
\end{tabular}


Citation: Ibrahim HS. Proteomic Translation of Chronic Granulomatous Disease (CGD). J Proteomics Computational Biol. $2017 ; 3(1)$ : 12.

ISSN: 2572-8679

\begin{tabular}{|c|c|c|c|c|c|c|c|}
\hline rs151344491 & Homo_sapiens/GRCh37.74 & G147R & G & $\mathrm{R}$ & -0.44 & 5 & Decrease \\
\hline rs151344492 & Homo_sapiens/GRCh37.74 & L546P & $\mathrm{L}$ & $\mathrm{P}$ & -1.32 & 5 & Decrease \\
\hline rs151344492 & Homo_sapiens/GRCh37.74 & L279P & $\mathrm{L}$ & $\mathrm{P}$ & -1.32 & 5 & Decrease \\
\hline rs151344492 & Homo_sapiens/GRCh37.74 & L514P & $\mathrm{L}$ & $\mathrm{P}$ & -1.32 & 5 & Decrease \\
\hline rs151344493 & Homo_sapiens/GRCh37.74 & S193F & $\mathrm{s}$ & $\mathrm{F}$ & -0.03 & 1 & Decrease \\
\hline rs151344493 & Homo_sapiens/GRCh37.74 & $\mathrm{S} 161 \mathrm{~F}$ & $\mathrm{~s}$ & $\mathrm{~F}$ & -0.03 & 1 & Decrease \\
\hline rs151344495 & Homo_sapiens/GRCh37.74 & L342Q & $\mathrm{L}$ & $Q$ & -1.81 & 8 & Decrease \\
\hline rs151344495 & Homo_sapiens/GRCh37.74 & L75Q & L & $\mathrm{Q}$ & -1.81 & 8 & Decrease \\
\hline rs151344495 & Homo_sapiens/GRCh37.74 & L310Q & $\mathrm{L}$ & $Q$ & -1.81 & 8 & Decrease \\
\hline rs151344496 & Homo_sapiens/GRCh37.74 & F205I & $\mathrm{F}$ & 1 & -0.84 & 8 & Decrease \\
\hline rs151344496 & Homo_sapiens/GRCh37.74 & F173I & $\mathrm{F}$ & 1 & -0.84 & 8 & Decrease \\
\hline rs151344497 & Homo_sapiens/GRCh37.74 & T178P & $\mathrm{T}$ & $\mathrm{P}$ & -0.38 & 5 & Decrease \\
\hline rs151344497 & Homo_sapiens/GRCh37.74 & $\mathrm{T} 146 \mathrm{P}$ & $\mathrm{T}$ & $\mathrm{P}$ & -0.38 & 5 & Decrease \\
\hline rs151344498 & Homo_sapiens/GRCh37.74 & Q231P & Q & $\mathrm{P}$ & -0.46 & 5 & Decrease \\
\hline rs151344498 & Homo_sapiens/GRCh37.74 & Q199P & $Q$ & $\mathrm{P}$ & -0.46 & 5 & Decrease \\
\hline rs200614534 & Homo_sapiens/GRCh37.74 & V407A & $\mathrm{V}$ & A & -2.02 & 10 & Decrease \\
\hline rs200614534 & Homo_sapiens/GRCh37.74 & V140A & $\mathrm{v}$ & A & -2.02 & 10 & Decrease \\
\hline rs200614534 & Homo_sapiens/GRCh37.74 & V375A & $\mathrm{v}$ & A & -2.02 & 10 & Decrease \\
\hline rs267606451 & Homo_sapiens/GRCh37.74 & S66F & $\mathrm{s}$ & $\mathrm{F}$ & 0.35 & 4 & Increase \\
\hline rs267606451 & Homo_sapiens/GRCh37.74 & S333F & s & $\mathrm{F}$ & 0.35 & 4 & Increase \\
\hline rs267606451 & Homo_sapiens/GRCh37.74 & S301F & $\mathrm{s}$ & $\mathrm{F}$ & 0.35 & 4 & Increase \\
\hline
\end{tabular}

\section{Methods}

Chronic granulomatous disease sequence (CGD) was retrieved from NCBI https://www.ncbi.nlm.nih.gov/projects/SNP/snp_ref. cgi?geneId=1536; rs141756032 [Homo sapiens], in chromosome X: 37804069; Gene: CYBB.

\section{Sift prediction}

(SIFT - Predict effects of non synonmous /missense variants) (http://sift.bii.a-star.edu.sg/) SIFT dbSNP 138 was selected from batch tools from SIFT Sorting Intolerant From Tolerant software to predict whether an amino acid substitution affects protein function, based on the sequence homology and the physical properties of amino acids. SIFT can be applied to naturally occurring non synonymous polymorphisms and laboratory-induced missense mutations.

\section{PolyPhen-2 (Polymorphism phenotyping v2)}

PolyPhen-2 prediction of functional effects of human nsSNPs (http://genetics.bwh.harvard.edu/pph2/index.shtml) was used to predict the impact of an amino acid substitution on the structure and function of a human protein using straight forward physical and comparative consideration [9-12].

\section{I-Mutant suite}

I mutant.3 (http://gpcr2.biocomp.unibo.it/cgi/predictors/ IMutant3.0/I-Mutant3.0.cgi) was used to predict the effect of single point protein mutation with disease association from Protein Sequence [13-15].

\section{SNPs and GO}

SNPs \& GO (http://snps.biofold.org/snps-and-go/snps-and-go. html) was used to predicting a disease associated variations by GO terms through SVM-based classifier to confirm SNPs results, by putting protein sequence, profile and functional information to give output inform of disease/neutral with RI \& scores [16-21].

\section{Results and Discussions}

141 out of 150 showed probably damaging by polyphen-2, while 3 showed possibly damaging and 6 were benign; that is why considered out of this study, all of them were considered deleterious by sift prediction including those showed benign predictions.

All those SNPs below showed deleterious, probably damaging with score predictions equal or slightly less than 1 according to sift \& polyphen-2 prediction sequential (see Table 1) rs137854585, rs137854586, rs137854587, rs137854589, rs137854591, rs137854593, rs137854594, rs137854595, rs137854596, rs139670417, rs141798777, rs146275471, rs151344453, rs151344454, rs151344456, rs151344457, rs151344458, rs151344459, rs151344460, rs151344462, rs151344465, rs151344466, rs151344467, rs151344468, rs151344469, rs151344470, rs151344471, rs151344472, rs151344473, rs151344474, rs151344475, rs151344477, rs151344478, rs151344479, rs151344480, rs151344481, rs151344482, rs151344484, rs151344485, rs151344486, rs151344487, rs151344488, rs151344489, rs151344490, rs151344491, rs151344492, rs151344493, rs151344495, rs151344496, rs151344497, rs151344498, rs200614534, rs267606451; except rs140677309 SNPs that showed both probably \& possibly damaging predictions.

\section{I mutant-3 prediction}

The total number of 150 SNPs showed 21 with increased protein activity while the remaining 129 showed decreased in protein activity, also the same SNPs had the same wide types but different mutant 
Citation: Ibrahim HS. Proteomic Translation of Chronic Granulomatous Disease (CGD). J Proteomics Computational Biol. 2017;3(1): 12.

ISSN: 2572-8679

Table 3: lllustrate SNPs \& GO predictions for CGD.

\begin{tabular}{|c|c|c|c|c|c|c|c|c|}
\hline SNP & Organism/Build & $\begin{array}{l}\text { Amino acid } \\
\text { change }\end{array}$ & $\begin{array}{l}\text { PhD-SNP } \\
\text { prediction }\end{array}$ & $\begin{array}{l}\text { PhD- } \\
\text { SNP IR }\end{array}$ & $\begin{array}{l}\text { PhD-SNP } \\
\text { probability }\end{array}$ & $\begin{array}{l}\text { SNPS \& GO } \\
\text { prediction }\end{array}$ & $\begin{array}{l}\text { SNPS \& } \\
\text { GO IR }\end{array}$ & $\begin{array}{l}\text { SNPS \& GO } \\
\text { probability }\end{array}$ \\
\hline rs137854585 & Homo_sapiens/GRCh37.74 & $\mathrm{P} 415 \mathrm{H}$ & Disease & 9 & 0.946 & Disease & 7 & 0.852 \\
\hline rs137854585 & Homo_sapiens/GRCh37.74 & $\mathrm{P} 148 \mathrm{H}$ & Disease & 9 & 0.945 & Disease & 7 & 0.851 \\
\hline rs137854585 & Homo_sapiens/GRCh37.74 & P383H & Disease & 9 & 0.946 & Disease & 7 & 0.852 \\
\hline rs137854585 & Homo_sapiens/GRCh37.74 & P415L & Disease & 9 & 0.952 & Disease & 8 & 0.897 \\
\hline rs137854585 & Homo_sapiens/GRCh37.74 & P148L & Disease & 9 & 0.95 & Disease & 8 & 0.896 \\
\hline rs137854585 & Homo_sapiens/GRCh37.74 & P383L & Disease & 9 & 0.951 & Disease & 8 & 0.896 \\
\hline rs137854586 & Homo_sapiens/GRCh37.74 & G389E & Disease & 8 & 0.917 & Disease & 8 & 0.882 \\
\hline rs137854586 & Homo_sapiens/GRCh37.74 & G122E & Disease & 8 & 0.917 & Disease & 8 & 0.882 \\
\hline rs137854586 & Homo_sapiens/GRCh37.74 & G357E & Disease & 8 & 0.918 & Disease & 8 & 0.883 \\
\hline rs137854586 & Homo_sapiens/GRCh37.74 & G389A & Disease & 7 & 0.833 & Disease & 6 & 0.819 \\
\hline rs137854586 & Homo_sapiens/GRCh37.74 & G122A & Disease & 7 & 0.834 & Disease & 6 & 0.819 \\
\hline rs137854586 & Homo_sapiens/GRCh37.74 & G357A & Disease & 7 & 0.835 & Disease & 6 & 0.821 \\
\hline rs137854587 & Homo_sapiens/GRCh37.74 & $\mathrm{H} 209 \mathrm{Y}$ & Disease & 9 & 0.974 & Disease & 8 & 0.89 \\
\hline rs137854587 & Homo_sapiens/GRCh37.74 & H177Y & Disease & 9 & 0.974 & Disease & 8 & 0.89 \\
\hline rs137854589 & Homo_sapiens/GRCh37.74 & C212Y & Disease & 8 & 0.894 & Disease & 1 & 0.563 \\
\hline rs137854589 & Homo_sapiens/GRCh37.74 & C244Y & Disease & 8 & 0.891 & Disease & 1 & 0.555 \\
\hline rs137854591 & Homo_sapiens/GRCh37.74 & H101R & Disease & 9 & 0.933 & Disease & 8 & 0.911 \\
\hline rs137854591 & Homo_sapiens/GRCh37.74 & H69R & Disease & 9 & 0.933 & Disease & 8 & 0.91 \\
\hline rs137854593 & Homo_sapiens/GRCh37.74 & D500G & Disease & 5 & 0.852 & Disease & 7 & 0.728 \\
\hline rs137854593 & Homo_sapiens/GRCh37.74 & D233G & Disease & 7 & 0.849 & Disease & 4 & 0.721 \\
\hline rs137854593 & Homo_sapiens/GRCh37.74 & D468G & Disease & 7 & 0.851 & Disease & 5 & 0.726 \\
\hline rs137854594 & Homo_sapiens/GRCh37.74 & H101Y & Disease & 9 & 0.93 & Disease & 7 & 0.862 \\
\hline rs137854594 & Homo_sapiens/GRCh37.74 & H69Y & Disease & 9 & 0.929 & Disease & 7 & 0.862 \\
\hline rs137854595 & Homo_sapiens/GRCh37.74 & $\mathrm{H} 36 \mathrm{~N}$ & Disease & 0 & 0.523 & Disease & 3 & 0.67 \\
\hline rs137854595 & Homo_sapiens/GRCh37.74 & $\mathrm{H} 303 \mathrm{~N}$ & Disease & 0 & 0.517 & Disease & 3 & 0.667 \\
\hline rs137854595 & Homo_sapiens/GRCh37.74 & $\mathrm{H} 271 \mathrm{~N}$ & Disease & 0 & 0.519 & Disease & 3 & 0.668 \\
\hline rs137854596 & Homo_sapiens/GRCh37.74 & P37R & Disease & 8 & 0.889 & Disease & 6 & 0.815 \\
\hline rs137854596 & Homo_sapiens/GRCh37.74 & P304R & Disease & 8 & 0.89 & Disease & 6 & 0.817 \\
\hline rs137854596 & Homo_sapiens/GRCh37.74 & P272R & Disease & 8 & 0.889 & Disease & 6 & 0.816 \\
\hline rs139670417 & Homo_sapiens/GRCh37.74 & $\mathrm{R} 229 \mathrm{H}$ & Disease & 3 & 0.635 & Neutral & 6 & 0.204 \\
\hline rs139670417 & Homo_sapiens/GRCh37.74 & R197H & Disease & 3 & 0.637 & Neutral & 6 & 0.205 \\
\hline rs140677309 & Homo_sapiens/GRCh37.74 & S258C & Neutral & 1 & 0.463 & Neutral & 2 & 0.403 \\
\hline rs140677309 & Homo_sapiens/GRCh37.74 & S525C & Neutral & 0 & 0.483 & Neutral & 2 & 0.416 \\
\hline rs140677309 & Homo_sapiens/GRCh37.74 & S493C & Neutral & 1 & 0.47 & Neutral & 2 & 0.412 \\
\hline rs141798777 & Homo_sapiens/GRCh37.74 & L75M & Neutral & 1 & 0.456 & Neutral & 7 & 0.133 \\
\hline rs141798777 & Homo_sapiens/GRCh37.74 & L43M & Neutral & 1 & 0.441 & Neutral & 7 & 0.129 \\
\hline rs146275471 & Homo_sapiens/GRCh37.74 & R198Q & Disease & 9 & 0.94 & Disease & 7 & 0.87 \\
\hline rs146275471 & Homo_sapiens/GRCh37.74 & R166Q & Disease & 9 & 0.94 & Disease & 7 & 0.87 \\
\hline rs151344453 & Homo_sapiens/GRCh37.74 & Y41D & Disease & 7 & 0.852 & Disease & 7 & 0.829 \\
\hline rs151344454 & Homo_sapiens/GRCh37.74 & C537R & Disease & 9 & 0.964 & Disease & 8 & 0.919 \\
\hline rs151344454 & Homo_sapiens/GRCh37.74 & C270R & Disease & 9 & 0.964 & Disease & 8 & 0.918 \\
\hline rs151344454 & Homo_sapiens/GRCh37.74 & C505R & Disease & 9 & 0.964 & Disease & 8 & 0.918 \\
\hline rs151344456 & Homo_sapiens/GRCh37.74 & R54S & Disease & 7 & 0.849 & Disease & 4 & 0.703 \\
\hline
\end{tabular}


Citation: Ibrahim HS. Proteomic Translation of Chronic Granulomatous Disease (CGD). J Proteomics Computational Biol. 2017;3(1): 12.

ISSN: 2572-8679

\begin{tabular}{|c|c|c|c|c|c|c|c|c|}
\hline rs151344456 & Homo_sapiens/GRCh37.74 & R22S & Disease & 8 & 0.899 & Disease & 6 & 0.781 \\
\hline rs151344457 & Homo_sapiens/GRCh37.74 & C59R & Disease & 8 & 0.902 & Disease & 7 & 0.852 \\
\hline rs151344457 & Homo_sapiens/GRCh37.74 & C27R & Disease & 8 & 0.899 & Disease & 7 & 0.848 \\
\hline rs151344458 & Homo_sapiens/GRCh37.74 & H119R & Disease & 9 & 0.937 & Disease & 8 & 0.909 \\
\hline rs151344458 & Homo_sapiens/GRCh37.74 & H87R & Disease & 9 & 0.937 & Disease & 8 & 0.908 \\
\hline rs151344459 & Homo_sapiens/GRCh37.74 & H209Q & Disease & 9 & 0.95 & Disease & 7 & 0.827 \\
\hline rs151344459 & Homo_sapiens/GRCh37.74 & $\mathrm{H} 177 \mathrm{Q}$ & Disease & 9 & 0.949 & Disease & 7 & 0.828 \\
\hline rs151344460 & Homo_sapiens/GRCh37.74 & $\mathrm{H} 222 \mathrm{~N}$ & Disease & 9 & 0.957 & Disease & 7 & 0.869 \\
\hline rs151344460 & Homo_sapiens/GRCh37.74 & H190N & Disease & 9 & 0.956 & Disease & 7 & 0.868 \\
\hline rs151344460 & Homo_sapiens/GRCh37.74 & $\mathrm{H} 222 \mathrm{Y}$ & Disease & 9 & 0.972 & Disease & 8 & 0.909 \\
\hline rs151344460 & Homo_sapiens/GRCh37.74 & H190Y & Disease & 9 & 0.971 & Disease & 8 & 0.908 \\
\hline rs151344462 & Homo_sapiens/GRCh37.74 & $\mathrm{H} 222 \mathrm{R}$ & Disease & 9 & 0.968 & Disease & 9 & 0.928 \\
\hline rs 151344462 & Homo_sapiens/GRCh37.74 & H190R & Disease & 9 & 0.967 & Disease & 9 & 0.928 \\
\hline rs151344465 & Homo_sapiens/GRCh37.74 & C212R & Disease & 7 & 0.874 & Disease & 1 & 0.544 \\
\hline rs151344465 & Homo_sapiens/GRCh37.74 & C244R & Disease & 7 & 0.871 & Disease & 1 & 0.537 \\
\hline rs151344466 & Homo_sapiens/GRCh37.74 & E42K & Disease & 4 & 0.683 & Disease & 4 & 0.714 \\
\hline rs151344466 & Homo_sapiens/GRCh37.74 & E309K & Disease & 4 & 0.704 & Disease & 5 & 0.727 \\
\hline rs151344466 & Homo_sapiens/GRCh37.74 & E277K & Disease & 4 & 0.702 & Disease & 5 & 0.725 \\
\hline rs 151344467 & Homo_sapiens/GRCh37.74 & G322E & Disease & 7 & 0.845 & Disease & 5 & 0.742 \\
\hline rs151344467 & Homo_sapiens/GRCh37.74 & G55E & Disease & 7 & 0.842 & Disease & 5 & 0.737 \\
\hline rs 151344467 & Homo_sapiens/GRCh37.74 & G290E & Disease & 7 & 0.844 & Disease & 5 & 0.74 \\
\hline rs151344468 & Homo_sapiens/GRCh37.74 & I325F & Disease & 5 & 0.735 & Neutral & 0 & 0.486 \\
\hline rs151344468 & Homo_sapiens/GRCh37.74 & $158 \mathrm{~F}$ & Disease & 5 & 0.732 & Neutral & 0 & 0.481 \\
\hline rs151344468 & Homo_sapiens/GRCh37.74 & $1293 \mathrm{~F}$ & Disease & 5 & 0.738 & Neutral & 0 & 0.488 \\
\hline rs151344469 & Homo_sapiens/GRCh37.74 & S66P & Disease & 9 & 0.938 & Disease & 8 & 0.878 \\
\hline rs151344469 & Homo_sapiens/GRCh37.74 & S333P & Disease & 9 & 0.94 & Disease & 8 & 0.88 \\
\hline rs151344469 & Homo_sapiens/GRCh37.74 & S301P & Disease & 9 & 0.939 & Disease & 8 & 0.879 \\
\hline rs151344470 & Homo_sapiens/GRCh37.74 & $\mathrm{P} 339 \mathrm{H}$ & Disease & 6 & 0.789 & Neutral & 0 & 0.477 \\
\hline rs151344470 & Homo_sapiens/GRCh37.74 & $\mathrm{P} 72 \mathrm{H}$ & Disease & 6 & 0.783 & Neutral & 1 & 0.471 \\
\hline rs151344470 & Homo_sapiens/GRCh37.74 & $\mathrm{P} 307 \mathrm{H}$ & Disease & 6 & 0.788 & Neutral & 0 & 0.476 \\
\hline rs1513444771 & Homo_sapiens/GRCh37.74 & R89P & Disease & 9 & 0.953 & Disease & 8 & 0.923 \\
\hline rs151344471 & Homo_sapiens/GRCh37.74 & R356P & Disease & 9 & 0.945 & Disease & 8 & 0.925 \\
\hline rs151344471 & Homo_sapiens/GRCh37.74 & R324P & Disease & 9 & 0.954 & Disease & 8 & 0.924 \\
\hline rs151344472 & Homo_sapiens/GRCh37.74 & M138R & Disease & 7 & 0.863 & Disease & 7 & 0.853 \\
\hline rs151344472 & Homo_sapiens/GRCh37.74 & M373R & Disease & 7 & 0.856 & Disease & 7 & 0.851 \\
\hline rs151344472 & Homo_sapiens/GRCh37.74 & M405R & Disease & 7 & 0.856 & Disease & 7 & 0.851 \\
\hline rs151344473 & Homo_sapiens/GRCh37.74 & G408R & Disease & 9 & 0.948 & Disease & 7 & 0.872 \\
\hline rs151344473 & Homo_sapiens/GRCh37.74 & G141R & Disease & 9 & 0.947 & Disease & 7 & 0.87 \\
\hline rs151344473 & Homo_sapiens/GRCh37.74 & G376R & Disease & 9 & 0.947 & Disease & 7 & 0.871 \\
\hline rs151344474 & Homo_sapiens/GRCh37.74 & G408E & Disease & 9 & 0.939 & Disease & 7 & 0.863 \\
\hline rs151344474 & Homo_sapiens/GRCh37.74 & G141E & Disease & 9 & 0.938 & Disease & 7 & 0.861 \\
\hline rs151344474 & Homo_sapiens/GRCh37.74 & G376E & Disease & 9 & 0.938 & Disease & 7 & 0.862 \\
\hline rs151344475 & Homo_sapiens/GRCh37.74 & S422P & Disease & 9 & 0.941 & Disease & 8 & 0.9 \\
\hline rs151344475 & Homo_sapiens/GRCh37.74 & S390P & Disease & 9 & 0.939 & Disease & 8 & 0.899 \\
\hline rs151344475 & Homo_sapiens/GRCh37.74 & S155P & Disease & 9 & 0.937 & Disease & 8 & 0.896 \\
\hline rs151344477 & Homo_sapiens/GRCh37.74 & W516C & Disease & 8 & 0.916 & Disease & 7 & 0.857 \\
\hline
\end{tabular}


Citation: Ibrahim HS. Proteomic Translation of Chronic Granulomatous Disease (CGD). J Proteomics Computational Biol. 2017;3(1): 12.

ISSN: 2572-8679

\begin{tabular}{|c|c|c|c|c|c|c|c|c|}
\hline rs151344477 & Homo_sapiens/GRCh37.74 & W249C & Disease & 8 & 0.91 & Disease & 7 & 0.856 \\
\hline rs151344477 & Homo_sapiens/GRCh37.74 & W484C & Disease & 8 & 0.916 & Disease & 7 & 0.856 \\
\hline rs151344478 & Homo_sapiens/GRCh37.74 & V534D & Disease & 9 & 0.936 & Disease & 8 & 0.892 \\
\hline rs151344478 & Homo_sapiens/GRCh37.74 & V267D & Disease & 9 & 0.935 & Disease & 8 & 0.891 \\
\hline rs151344478 & Homo_sapiens/GRCh37.74 & V502D & Disease & 9 & 0.936 & Disease & 8 & 0.892 \\
\hline rs151344479 & Homo_sapiens/GRCh37.74 & R54M & Disease & 7 & 0.827 & Disease & 2 & 0.595 \\
\hline rs151344479 & Homo_sapiens/GRCh37.74 & $\mathrm{R} 22 \mathrm{M}$ & Disease & 7 & 0.862 & Disease & 3 & 0.668 \\
\hline rs151344480 & Homo_sapiens/GRCh37.74 & A55D & Disease & 8 & 0.918 & Disease & 7 & 0.863 \\
\hline rs151344480 & Homo_sapiens/GRCh37.74 & A23D & Disease & 9 & 0.935 & Disease & 8 & 0.901 \\
\hline rs151344481 & Homo_sapiens/GRCh37.74 & A57E & Disease & 8 & 0.924 & Disease & 6 & 0.798 \\
\hline rs151344481 & Homo_sapiens/GRCh37.74 & A25E & Disease & 8 & 0.923 & Disease & 6 & 0.798 \\
\hline rs151344482 & Homo_sapiens/GRCh37.74 & H209R & Disease & 9 & 0.974 & Disease & 8 & 0.904 \\
\hline rs151344482 & Homo_sapiens/GRCh37.74 & H177R & Disease & 9 & 0.974 & Disease & 8 & 0.904 \\
\hline rs151344484 & Homo_sapiens/GRCh37.74 & H338Y & Disease & 6 & 0.805 & Disease & 6 & 0.803 \\
\hline rs151344484 & Homo_sapiens/GRCh37.74 & H71Y & Disease & 6 & 0.804 & Disease & 6 & 0.802 \\
\hline rs151344484 & Homo_sapiens/GRCh37.74 & H306Y & Disease & 6 & 0.804 & Disease & 6 & 0.803 \\
\hline rs151344485 & Homo_sapiens/GRCh37.74 & S312F & Disease & 7 & 0.871 & Disease & 5 & 0.768 \\
\hline rs151344485 & Homo_sapiens/GRCh37.74 & S344F & Disease & 7 & 0.872 & Disease & 5 & 0.769 \\
\hline rs151344485 & Homo_sapiens/GRCh37.74 & S77F & Disease & 7 & 0.856 & Disease & 5 & 0.752 \\
\hline rs151344486 & Homo_sapiens/GRCh37.74 & L420P & Disease & 9 & 0.958 & Disease & 8 & 0.89 \\
\hline rs151344486 & Homo_sapiens/GRCh37.74 & L153P & Disease & 9 & 0.959 & Disease & 8 & 0.892 \\
\hline rs151344486 & Homo_sapiens/GRCh37.74 & L388P & Disease & 9 & 0.958 & Disease & 8 & 0.891 \\
\hline rs151344487 & Homo_sapiens/GRCh37.74 & W516R & Disease & 8 & 0.913 & Disease & 7 & 0.858 \\
\hline rs151344487 & Homo_sapiens/GRCh37.74 & W249R & Disease & 9 & 0.91 & Disease & 7 & 0.856 \\
\hline rs151344487 & Homo_sapiens/GRCh37.74 & W484R & Disease & 9 & 0.913 & Disease & 7 & 0.859 \\
\hline rs151344488 & Homo_sapiens/GRCh37.74 & C59W & Disease & 8 & 0.879 & Disease & 5 & 0.785 \\
\hline rs151344488 & Homo_sapiens/GRCh37.74 & C27W & Disease & 7 & 0.869 & Disease & 5 & 0.745 \\
\hline rs151344489 & Homo_sapiens/GRCh37.74 & T307P & Disease & 5 & 0.747 & Neutral & 4 & 0.288 \\
\hline rs151344489 & Homo_sapiens/GRCh37.74 & T40P & Disease & 5 & 0.747 & Neutral & 5 & 0.287 \\
\hline rs151344489 & Homo_sapiens/GRCh37.74 & T275P & Disease & 5 & 0.75 & Neutral & 4 & 0.29 \\
\hline rs151344490 & Homo_sapiens/GRCh37.74 & L505R & Disease & 8 & 0.894 & Disease & 7 & 0.864 \\
\hline rs151344490 & Homo_sapiens/GRCh37.74 & L238R & Disease & 8 & 0.898 & Disease & 7 & 0.866 \\
\hline rs151344490 & Homo_sapiens/GRCh37.74 & L473R & Disease & 8 & 0.894 & Disease & 7 & 0.864 \\
\hline rs151344491 & Homo_sapiens/GRCh37.74 & G179R & Disease & 9 & 0.955 & Disease & 8 & 0.884 \\
\hline rs151344491 & Homo_sapiens/GRCh37.74 & G147R & Disease & 9 & 0.955 & Disease & 8 & 0.883 \\
\hline rs151344492 & Homo_sapiens/GRCh37.74 & L546P & Disease & 6 & 0.8 & Disease & 4 & 0.689 \\
\hline rs151344492 & Homo_sapiens/GRCh37.74 & L279P & Disease & 6 & 0.802 & Disease & 4 & 0.689 \\
\hline rs151344492 & Homo_sapiens/GRCh37.74 & L514P & Disease & 6 & 0.8 & Disease & 4 & 0.689 \\
\hline rs151344493 & Homo_sapiens/GRCh37.74 & S193F & Disease & 7 & 0.867 & Disease & 5 & 0.768 \\
\hline rs151344493 & Homo_sapiens/GRCh37.74 & S161F & Disease & 7 & 0.866 & Disease & 5 & 0.767 \\
\hline rs151344495 & Homo_sapiens/GRCh37.74 & L342Q & Disease & 6 & 0.775 & Disease & 5 & 0.747 \\
\hline rs151344495 & Homo_sapiens/GRCh37.74 & L75Q & Disease & 6 & 0.779 & Disease & 5 & 0.747 \\
\hline rs151344495 & Homo_sapiens/GRCh37.74 & L310Q & Disease & 6 & 0.775 & Disease & 5 & 0.746 \\
\hline rs151344496 & Homo_sapiens/GRCh37.74 & F205I & Disease & 9 & 0.971 & Disease & 8 & 0.913 \\
\hline rs151344496 & Homo_sapiens/GRCh37.74 & F173I & Disease & 9 & 0.971 & Disease & 8 & 0.914 \\
\hline rs151344497 & Homo_sapiens/GRCh37.74 & T178P & Disease & 9 & 0.952 & Disease & 8 & 0.859 \\
\hline
\end{tabular}


Citation: Ibrahim HS. Proteomic Translation of Chronic Granulomatous Disease (CGD). J Proteomics Computational Biol. $2017 ; 3(1): 12$.

ISSN: 2572-8679

\begin{tabular}{|l|l|l|l|l|l|l|c|c|}
\hline rs151344497 & Homo_sapiens/GRCh37.74 & T146P & Disease & 9 & 0.952 & Disease & 7 & 0.857 \\
\hline rs151344498 & Homo_sapiens/GRCh37.74 & Q231P & Disease & 8 & 0.882 & Neutral & 1 & 0.428 \\
\hline rs151344498 & Homo_sapiens/GRCh37.74 & Q199P & Disease & 8 & 0.882 & Neutral & 1 & 0.428 \\
\hline rs200614534 & Homo_sapiens/GRCh37.74 & V407A & Disease & 6 & 0.808 & Disease & 1 & 0.541 \\
\hline rs200614534 & Homo_sapiens/GRCh37.74 & V140A & Disease & 6 & 0.808 & Disease & 1 & 0.539 \\
\hline rs200614534 & Homo_sapiens/GRCh37.74 & V375A & Disease & 6 & 0.808 & Disease & 1 & 0.541 \\
\hline rs267606451 & Homo_sapiens/GRCh37.74 & S66F & Disease & 9 & 0.938 & Disease & 8 & 0.878 \\
\hline rs267606451 & Homo_sapiens/GRCh37.74 & S333F & Disease & 8 & 0.918 & Disease & 7 & 0.845 \\
\hline rs267606451 & Homo_sapiens/GRCh37.74 & S301F & Disease & 8 & 0.917 & Disease & 7 & 0.853 \\
\hline
\end{tabular}

types, instead of these some of them shared the same mutant types; as illustrated in Table 2 below.

\section{SNPs \& GO predictions}

The total numbers of 126 SNPs were showed disease prediction by both PhD-SNP prediction and SNPS \& GO prediction they are: rs137854585, rs137854586, rs137854587, rs137854589, rs137854591, rs137854593, rs137854594, rs137854595, rs137854596, rs139670417, rs141798777, rs146275471, rs151344453, rs151344454, rs151344456, rs151344457, rs151344458, rs151344459, rs151344460, rs151344462, rs151344465, rs151344466, rs151344467, rs151344468, rs151344469, rs151344470, rs151344471, rs151344472, rs151344473, rs151344474,rs151344475, rs151344477, rs151344478, rs151344479, rs151344480, rs151344481, rs151344482, rs151344484, rs151344485, rs151344486, rs151344487, rs151344488, rs151344489, rs151344490, rs151344491, rs151344492, rs151344493, rs151344495, rs151344496, rs151344497, rs151344498, rs200614534, rs267606451, except 13 SNPs they were showed both diseases \& neutrals according to PhDSNP prediction and SNPS \& GO prediction sequential; they are: rs139670417, rs151344468, rs151344470, rs151344489, rs151344498 while 5 SNPs were showed neutral for both PhD-SNP prediction and SNPS \& GO prediction they are; rs140677309 and rs141798777 (see Table 3).

\section{Conclusions}

The output of this study were explained and confirmed the damaging effect of those selected CGD SNPs. Although due to some protein ID problems in ENSP00000441958 \&ENSP00000441896; I used UPI00020654B0 \& UPI00020654AF instead of them; which were suggested by uniprot web site to complete polyphen-2 prediction, also I faced error prediction with some sequences in meta-snps prediction, that is explains why I exclude them from this study, so on I suggest to check this program again to know where is the problem.

\section{References}

1. Chronic Granulomatous Disease (CGD).

2. (2016) Chronic granulomatous disease. NIH.U.S. National library of medicine. Genetics Home Reference.

3. (2017) Chronic granulomatous disease (CGD). American Academy of Allergy Asthma \& Immunology.

4. (2017) Chronic granulomatous disease. MedlinePlus

5. (2016) Chronic granulomatous disease. From Wikipedia, the free encyclopedia.

6. Dinauer MC (2016) Chronic granulomatous disease. National Organization for Rare Disorders.
7. IDF (2016) Chronic granulomatous disease and other phagocytic cell disorders. Immune Deficiency Foundation.

8. Nowicki RJ, Elston DM (2016) Chronic granulomatous disease. Medscape.

9. Adzhubei IA, Schmidt S, Peshkin L, Ramensky VE, Gerasimova A, et al. (2010) A method and server for predicting damaging missense mutations. Nat Methods 7: 248-249.

10. Adzhubei I, Jordan DM, Sunyaev SR (2013) Predicting functional effect of human missense mutations using PolyPhen-2. Curr Protoc Hum Genet 7 : Unit 7.20.

11. Ramensky V, Bork P, Sunyaev S (2002) Human non-synonymous SNPs: server and survey. Nucleic Acids Res 30: 3894-3900.

12. Sunyaev SR, Eisenhaber F, Rodchenkov IV, Eisenhaber B, Tumanyan VG et al. (1999) PSIC: profile extraction from sequence alignments with positionspecific counts of independent observations. Protein Eng 12: 387-394.

13. Capriotti E, Fariselli P, Casadio R (2005) I-Mutant2.0: predicting stability changes upon mutation from the protein sequence or structure. Nucleic Acids Res 33: W306-W310

14. Capriotti E, Fariselli P, Calabrese R, Casadio R (2005) Predicting protein stability changes from sequences using support vector machines. Bioinformatics 21 Suppl 2: ii54-ii58.

15. Capriotti E, Calabrese R, Casadio R (2006) Predicting the insurgence of human genetic diseases associated to single point protein mutations with Support Vector Machines and evolutionary information. Bioinformatics 22 : 2729-2734.

16. Calabrese R, Capriotti E, Fariselli P, Martelli PL, Casadio R (2009) Functional annotations improve the predictive score of human disease-related mutations in proteins. Hum Mutat 30: 1237-1244.

17. Altschul SF, Madden TL, Schaffer AA, Zhang J, Zhang Z, et al. (1997) Gapped BLAST and PSI-BLAST: a new generation of protein database search programs. Nucleic Acids Res 25: 3389-3402.

18. Thomas PD, Kejariwal A (2004) Coding single-nucleotide polymorphisms associated with complex vs. Mendelian disease: Evolutionary evidence for differences in molecular effects. Proc Natl Acad Sci U S A 101: 15398-15403.

19. Capriotti E, Altman RB (2011) Improving the prediction of disease-related variants using protein three-dimensional structure. BMC Bioinformatics 12: S3.

20. Kabsch W, Sander C (1983) Dictionary of protein secondary structure: pattern recognition of hydrogen-bonded and geometrical features. Biopolymers 22 : 2577-2637

21. Thusberg J, Olatubosun A, Vihinen M (2011) Performance of mutation pathogenicity prediction methods on missense variants. Hum Mutat 32: 358368.

\section{Acknowledgements}

The author would like to thanks Allah, her family for always supporting her, Bio-Nile center members. 